\title{
VALIDACIÓN DE UN MÉTODO POR CROMATOGRAFÍA LÍQUIDA DE ALTA RESOLUCIÓN PARA LA CUANTIFICACIÓN DE SENÓSIDOS EN TABLETAS DE CHOCOLATE
}

\author{
Jhonnel Samaniego*a, Jenny Huerta ${ }^{\mathrm{a}}$, Miguel Inocente ${ }^{\mathrm{a}}$, Juan Obregón ${ }^{\mathrm{b}}$, Maricela López ${ }^{\mathrm{b}}$
}

\section{RESUMEN}

La industria farmacéutica, para el desarrollo de un nuevo producto, necesita utilizar un método analítico que permita cuantificar, de forma rápida y confiable, el principio activo. En la validación, se determina si el método es fiable y reproducible, por ello, en el presente trabajo, se valida un método para cuantificar senósidos en tabletas por cromatografía líquida de alta resolución, el tipo de investigación fue descriptivo con muestreo aleatorio simple. Las condiciones cromatográficas empleadas son: columna L11 (Phenyl) $15 \mathrm{~cm} \mathrm{x} \mathrm{4,6} \mathrm{mm} \mathrm{x}$ $5 \mu \mathrm{m}$, temperatura del horno a $40{ }^{\circ} \mathrm{C}$, longitud de onda a $274 \mathrm{~nm}$, fase móvil con gradiente de flujo que oscila entre 0,4 y $1,5 \mathrm{~mL} /$ minuto y de solvente, compuesta por buffer fosfato $\mathrm{pH} 3,5$ y Acetonitrilo, volumen de inyección $40 \mu \mathrm{L}$. Los parámetros de validación evaluados confirmaron la especificidad, la linealidad a concentraciones de 0,$07 ; 0,11 ; 0,15 ; 0,19$ y 0,23 $\mathrm{mg} / \mathrm{mL}$ con un coeficiente de correlación de 0,9996 , la precisión con coeficiente de variación menor a $2 \%$, la exactitud del $98,6 \%$ y la robustez después de 24 horas con C.V. $(\%) \leq 2 \%$. Se determina que el método propuesto es apto para la cuantificación de senósidos en tabletas de chocolate.

Palabras clave: validación; cromatografía líquida de alta resolución; senósidos.

\section{VALIDATION OF A METHOD BY HIGH PERFORMANCE LIQUID CHROMATOGRAPHY FOR THE QUANTIFICATION OF SENNOSIDES IN CHOCOLATE BARS}

\begin{abstract}
To develop a new product pharmaceutical industries, use analytical methods to quantify the active principle fast and reliable. The validation was determined if the method is reliable and reproducible, for this reason the present work validated an analytical method by high resolution liquid chromatography to quantify sennosides in chocolate bars, the type of research was descriptive with simple random sampling. The chromatographic conditions

\footnotetext{
a Facultad de Ciencias de la Salud, Universidad María Auxiliadora, Av. Canto Bello 431, San Juan de Lurigancho, Lima 15408, Perú. jhonnel.samaniego@uma.edu.pe

b Facultad de Ciencias de la Salud, Universidad Nacional San Cristóbal de Huamanga, Ayacucho, Perú.
} 
were: column L11 (Phenyl) $15 \mathrm{~cm}$ x $4.6 \mathrm{~mm}$ x $5 \mu \mathrm{m}$, oven temperature at $40{ }^{\circ} \mathrm{C}$, wavelength at $274 \mathrm{~nm}$, mobile phase with a flow gradient ranging from 0.4 to $1,5 \mathrm{~mL} /$ minute and solvent, composed to phosphate buffer $\mathrm{pH} 3.5$ and Acetonitrile, injection volume $40 \mu \mathrm{L}$. The evaluated validation parameters confirmed specificity, linearity to concentrations of 0.07 ; $0.11 ; 0.15 ; 0.19$ and $0.23 \mathrm{mg} / \mathrm{mL}$ with a correlation coefficient of 0.9996 , precision with a coefficient of variation less than $2 \%$, accuracy of $98.6 \%$ and robustness after 24 hours with C.V. $(\%) \leq 2 \%$. The proposed method that the proposed method is suitable for the quantification of sennosides in chocolate bars.

Key words: validation; high performance liquid chromatography; sennosides.

\section{INTRODUCCIÓN}

En el desarrollo de un producto farmacéutico es necesario utilizar un método analítico específico que permita cuantificar el principio activo en una formulación, y para asegurar su confiabilidad es necesario validarlo ${ }^{1,2,3}$. Esta validación consiste en determinar diversos parámetros, que van a depender de la categoría a la que pertenezcan 4,5. Diversos organismos reguladores como la Food and Drug Administration (FDA), Agencia Nacional de Vigilancia Sanitaria (ANVISA), Agencia Española de Medicamentos y Productos Sanitarios (AEMPS); así como las referencias The United States Pharmacopeia (USP), International Conference on Harmonisation (ICH) y Asociación Española de Farmacéuticos de la Industria (AEFI), exigen y recomiendan la validación como requisito imprescindible para el cumplimiento de la Buenas Prácticas de Laboratorio (BPL)4. La cromatografía líquida de alta resolución (HPLC) posee diversas aplicaciones y, por ser una técnica automatizada, permite analizar de forma rápida y eficiente la separación, cuantificación y detección de las muestras por medio de la identificación de picos en los cromatogramas, que se pueden optimizar mediante el ajuste de múltiples variables en el método ( $\mathrm{pH}$ de la fase móvil, concentración del buffer, flujo, temperatura de la columna, longitud de onda del detector, etc. $)^{6,7,8,9,10,11}$. Al obtener un método rápido y confiable, puede ser aplicado en uso rutinario en el análisis de control de calidad y determinación de la estabilidad del medicamento ${ }^{12,13,14,15,16}$. Las plantas medicinales, y su uso en salud complementaria, son tan antiguas como la aparición de la especie humana, siendo parte de las prácticas de atención familiar y comunitaria. En la actualidad, se está volviendo a utilizar las plantas medicinales en la salud y cabe resaltar que los senósidos son un laxante natural de origen vegetal para el tratamiento de la constipación leve provocada por malos hábitos alimentarios, falta de ejercicio e inadecuada ingesta de fibra natural ${ }^{17,18,19}$.

En la presente investigación se tiene como objetivo validar un método analítico por cromatografía líquida de alta resolución para la cuantificación de senósidos en tabletas y, para ello, establecer parámetros de validación que permitan tener la confiabilidad para el uso rutinario en la determinación de calidad de medicamentos. 


\section{PARTE EXPERIMENTAL}

Reactivos: Se utilizó potasio dihidrogenofosfato de Merck (Darmstadt, Alemania), ácido orto-fosfórico $85 \%$ de Merck (Darmstadt, Alemania) y acetonitrilo de Merck (Darmstadt, Alemania).

Condiciones cromatográficas: Como fase móvil, se utilizaron dos soluciones: Solución A (Buffer fosfato $\mathrm{pH} 3,5$ ) disolviendo $6,8 \mathrm{~g}$ de potasio dihidrogenofosfato, por cada 1000 $\mathrm{mL}$ de agua purificada, se ajustó a $\mathrm{pH} 3,5$ con ácido orto-fosfórico $85 \%$ y la Solución B: acetonitrilo. La columna cromatográfica utilizada fue L11 Fenilo de $150 \mathrm{~mm}$ x 4,6 mm x 5 $\mu \mathrm{m}$, el detector UV a $274 \mathrm{~nm}$, la temperatura del horno a $40^{\circ} \mathrm{C}$, el volumen de inyección de $40 \mu \mathrm{L}$, la velocidad de flujo de $0,4 \mathrm{~mL} /$ minuto, el tiempo de retención del pico principal es de 15,0 minutos y el tiempo de cada corrida es de 50 minutos.

Tabla 1. Elución en gradiente de la fase móvil de solución A y solución B.

\begin{tabular}{cccc}
\hline Tiempo & $\begin{array}{c}\text { Solución } \\
\text { A }\end{array}$ & $\begin{array}{c}\text { Solución } \\
\text { B }\end{array}$ & Flujo \\
\hline 0,0 & 92,0 & 8,0 & $0,4 \mathrm{~mL} / \mathrm{min}$. \\
5,0 & 92,0 & 8,0 & $0,4 \mathrm{~mL} / \mathrm{min}$. \\
15,0 & 92,0 & 8,0 & $0,4 \mathrm{~mL} / \mathrm{min}$. \\
22,0 & 92,0 & 8,0 & $0,4 \mathrm{~mL} / \mathrm{min}$. \\
23,0 & 92,0 & 8,0 & $1,0 \mathrm{~mL} / \mathrm{min}$. \\
24,0 & 92,0 & 8,0 & $1,5 \mathrm{~mL} / \mathrm{min}$. \\
33,0 & 92,0 & 8,0 & $1,5 \mathrm{~mL} / \mathrm{min}$. \\
34,0 & 92,0 & 8,0 & $1,0 \mathrm{~mL} / \mathrm{min}$. \\
35,0 & 92,0 & 8,0 & $0,4 \mathrm{~mL} / \mathrm{min}$. \\
50,0 & 92,0 & 8,0 & $0,4 \mathrm{~mL} / \mathrm{min}$. \\
\hline
\end{tabular}

Preparación de estándar: Se utilizó un estándar de referencia de senósidos en solución buffer $\mathrm{pH} 3,5$ a una concentración aproximada de $0,15 \mathrm{mg} / \mathrm{mL}$ que luego se filtró por una membrana de $0,45 \mu \mathrm{m}$.

Preparación de la muestra: Se refrigeraron las tabletas de chocolate por 1 hora para luego fraccionarlas, se pesó una cantidad de muestra equivalente a $15 \mathrm{mg}$ de senósidos en un matraz volumétrico y se le agregó $25 \mathrm{~mL}$ de éter de petróleo, luego se llevó a calentar en baño maría de $50{ }^{\circ} \mathrm{C}$, hasta completa dispersión. Se adicionó $100 \mathrm{~mL}$ de solución buffer fosfato $\mathrm{pH} 3,5$; se calentó en baño maría a $50{ }^{\circ} \mathrm{C}$ y se agitó de manera intermitente durante 30 minutos, se filtró de inmediato, a través de papel Whatman, (40) se dejó enfriar la solución y luego se filtró por membrana de $0,45 \mu \mathrm{m}$, obteniendo una concentración aproximada de $0,15 \mathrm{mg} / \mathrm{mL}$ de senósidos. 


\section{Determinación de algunos parámetros de validación}

Especificidad: Para la determinación de posibles interferencias, se analizó el placebo con el principio activo al $100 \%$ y se determinó el grado de interferencia con respecto al análisis del principio activo, con y sin placebo, debiendo obtener resultados $\pm 2 \%$ del teórico. En la determinación de interferencias de productos de degradación, se expusieron tanto placebo muestra y principio activo a diferentes tipos de estrés como luz UV, calor a $80{ }^{\circ} \mathrm{C}$ por 24 horas, hidrólisis ácida por 2 horas a $80^{\circ} \mathrm{C}$, hidrólisis alcalina por 2 horas a $80^{\circ} \mathrm{C}$, oxidación con peróxido al $30 \%$ por 2 horas.

Linealidad e intervalo: Para evaluar la linealidad del sistema se prepararon 5 concentraciones en el rango de $50 \%, 75 \%, 100 \%, 125 \%$ y $150 \%$. En la linealidad del método se prepararon soluciones de placebo enriquecida con principio activo en tres niveles $75 \%, 100 \%$ y $125 \% 5$.

Exactitud: Para evaluar este parámetro se utilizaron las mismas muestras de la linealidad del método 5 .

Precisión: Para la repetibilidad se analizaron 6 muestras independientes. En la precisión intermedia, un segundo analista lo evaluó usando el mismo método analítico en diferentes equipos cromatográficos.

Robustez: Para ello se consideró tres muestras del análisis de repetibilidad como análisis inicial y ellas permanecieron 24 horas a temperatura ambiente y luego se volvió a analizar con un estándar recientemente preparado.

\section{RESULTADOS Y DISCUSIÓN}

En la prueba de especificidad se evaluó las posibles interferencias originadas en la muestra, debido al estrés forzado sometido por fotolisis y termólisis, encontrándose los siguientes resultados, según tabla 2 . 
Tabla 2. Especificidad de posibles interferentes en senósidos en tabletas de chocolate.

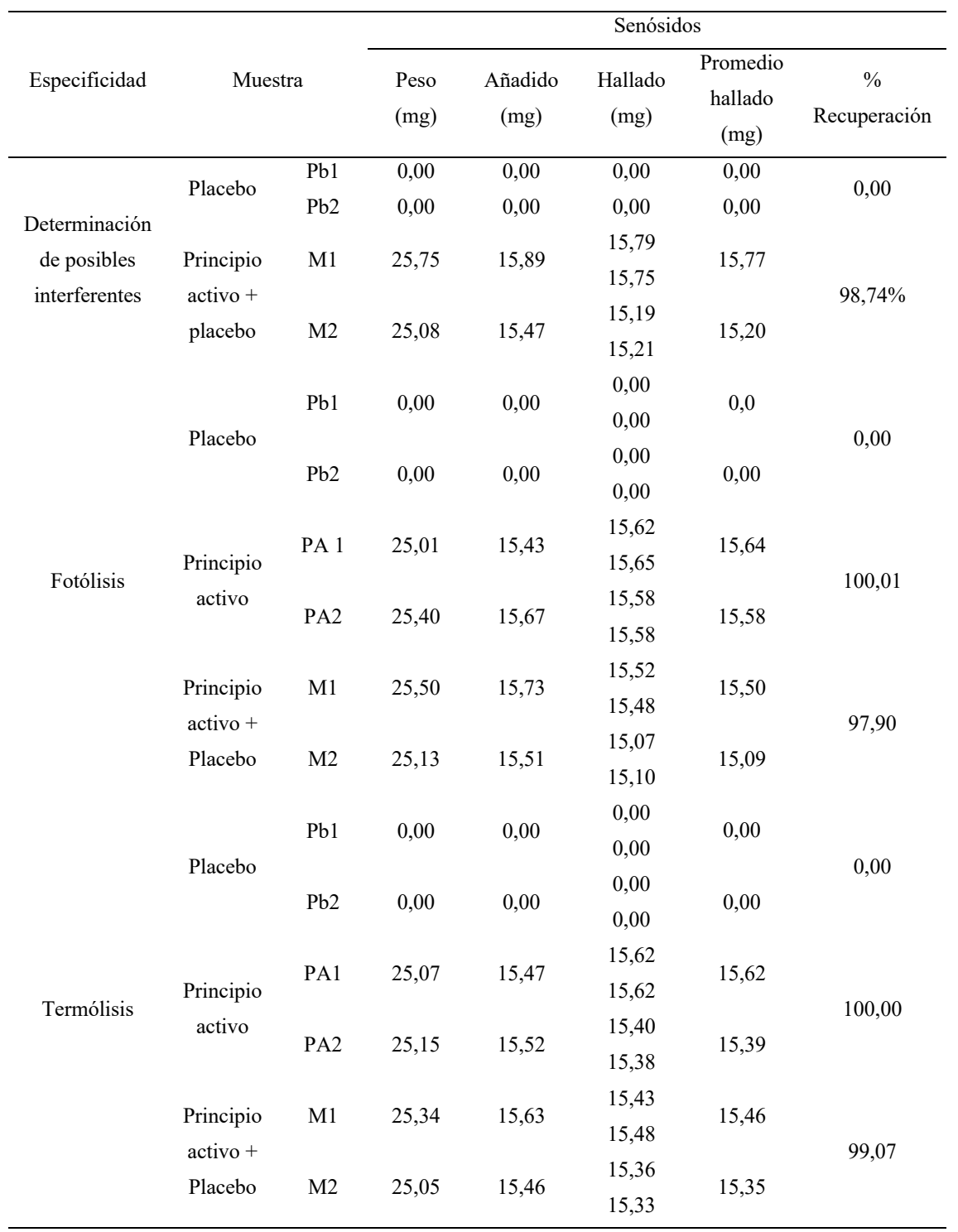

$\mathrm{Pb}$ : placebo $\mathrm{PA}$ : principio activo $\mathrm{M}$ : muestra

En la tabla 3, se muestran los resultados del ensayo de Especificidad en Senósidos tabletas de chocolate sometidas a estrés forzado por hidrólisis acida, hidrólisis alcalina y estrés oxidativo. 
Tabla 3. Especificidad de hidrólisis ácida, alcalina y oxidativa.

\begin{tabular}{|c|c|c|c|c|c|c|c|}
\hline \multirow[b]{2}{*}{ Especificidad } & \multirow{2}{*}{\multicolumn{2}{|c|}{ Muestra }} & \multicolumn{5}{|c|}{ Senósidos } \\
\hline & & & \multirow{3}{*}{\begin{tabular}{|c} 
Peso (mg) \\
0,00
\end{tabular}} & \multirow{3}{*}{$\begin{array}{c}\begin{array}{c}\text { Añadido } \\
(\mathrm{mg})\end{array} \\
0,00\end{array}$} & \multirow{3}{*}{$\begin{array}{c}\begin{array}{c}\text { Hallado } \\
\text { (mg) }\end{array} \\
0,00\end{array}$} & \multirow{3}{*}{$\begin{array}{c}\begin{array}{c}\text { Promedio } \\
\text { hallado }(\mathrm{mg})\end{array} \\
0,00\end{array}$} & \multirow{4}{*}{$\begin{array}{c}\% \\
\text { Recuperación } \\
0,00\end{array}$} \\
\hline \multirow{11}{*}{ Hidrólisis ácida } & \multirow{3}{*}{ Placebo } & $\mathrm{Pb} 1$ & & & & & \\
\hline & & 101 & & & & & \\
\hline & & $\mathrm{Pb} 2$ & 0,00 & 0,00 & 0,00 & 0,00 & \\
\hline & \multirow{4}{*}{$\begin{array}{l}\text { Principio } \\
\text { activo }\end{array}$} & \multirow{2}{*}{ PA1 } & \multirow{2}{*}{25,84} & \multirow{2}{*}{15,94} & 11,63 & \multirow{2}{*}{11,62} & \multirow{4}{*}{71,97} \\
\hline & & & & & 11,61 & & \\
\hline & & \multirow{2}{*}{ PA2 } & \multirow{2}{*}{24,92} & \multirow{2}{*}{15,38} & 10,92 & \multirow{2}{*}{10,92} & \\
\hline & & & & & 10,91 & & \\
\hline & \multirow{4}{*}{$\begin{array}{l}\text { Principio } \\
\text { activo }+ \\
\text { placebo }\end{array}$} & \multirow{2}{*}{ M1 } & \multirow{2}{*}{25,01} & \multirow{2}{*}{15,43} & 11,55 & \multirow{2}{*}{11,50} & \multirow{4}{*}{75,02} \\
\hline & & & & & 11,45 & & \\
\hline & & \multirow{2}{*}{ M2 } & 2574 & 1588 & 11,99 & 1199 & \\
\hline & & & 25,14 & 15,88 & 11,98 & 11,99 & \\
\hline & & $\mathrm{Pb} 1$ & 000 & 0.00 & 0,00 & 00 & \\
\hline & Placebo & & & & 0,00 & & 0,00 \\
\hline & РТасеоo & $\mathrm{Ph} 2$ & 000 & 000 & 0,00 & 000 & \\
\hline & & 102 & 0,00 & 0,00 & 0,00 & 0,00 & \\
\hline & & & & & 13,02 & & \\
\hline Hidrólisis & Principio & PA 1 & 25,08 & 15,47 & 12,98 & 13,00 & 8374 \\
\hline alcalina & activo & & & & 12,90 & & 05,14 \\
\hline & & PA2 & 25,05 & 15,46 & 12,89 & 12,90 & \\
\hline & & & & & 13,23 & & \\
\hline & Principio & M1 & 25,03 & 15,44 & 13,21 & 13,22 & \\
\hline & activo + & בר & 2503 & 15.44 & 12,94 & 1207 & 84,70 \\
\hline & 1 1acevo & 1012 & 25,03 & 15,44 & 12,99 & 12,91 & \\
\hline & & $\mathrm{Pb} 1$ & 0,00 & 0.00 & 0,00 & 0,00 & \\
\hline & Placebo & & & & 0,00 & & 0.00 \\
\hline & & $\mathrm{Pb} 2$ & 000 & 0.00 & 0,00 & 000 & \\
\hline & & PDZ & 0,00 & 0,00 & 0,00 & 0,00 & \\
\hline & & PA1 & 25,15 & 15,52 & 11,37 & 11,34 & \\
\hline Oxidación & Principio & PAI & 25,15 & 15,52 & 11,31 & 11,34 & 7237 \\
\hline Uxidacion & activo & PA2 & 25.23 & 15,57 & 11,15 & 11,16 & \\
\hline & & PA2 & 25,23 & 15,51 & 11,16 & 11,10 & \\
\hline & & & & & 7,03 & & \\
\hline & Principio & MI & 25,16 & 15,52 & 7,01 & 7,02 & \\
\hline & $\begin{array}{l}\text { activo + } \\
\text { Placebo }\end{array}$ & M2 & 25.45 & 1570 & 7,24 & 712 & 45,25 \\
\hline & & 1012 & 25,45 & 15,10 & 6,99 & 1,12 & \\
\hline
\end{tabular}

$\mathrm{Pb}$ : placebo PA: principio activo $\mathrm{M}$ : muestra

En la figura 1, se reflejan los resultados obtenidos al evaluar el parámetro de Linealidad del sistema tomando las 5 concentraciones, con el siguiente resultado aplicando la ecuación de la recta para la verificación de la confiabilidad del sistema. 


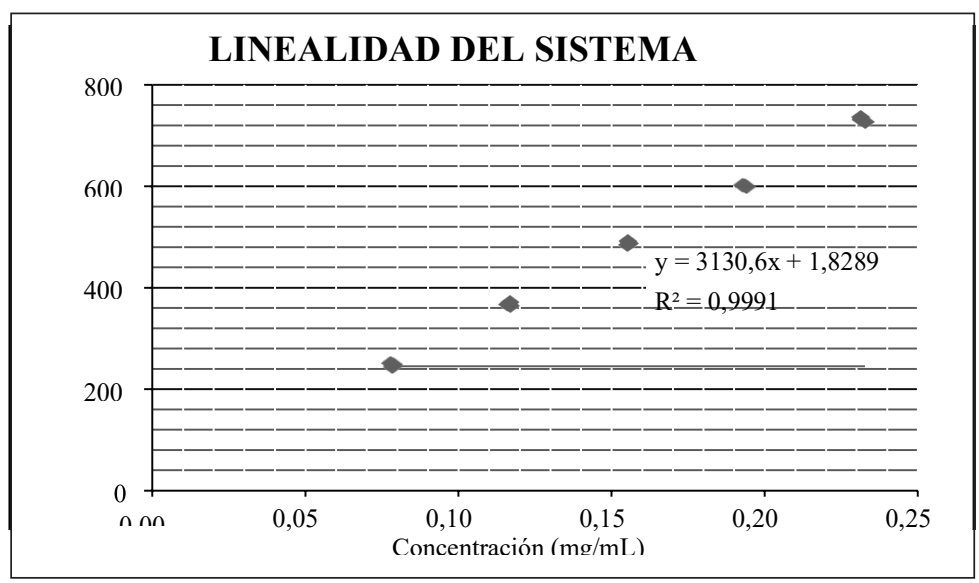

Figura 1. Área versus concentración de linealidad del sistema de senósidos en tabletas de chocolate.

En la figura 2, se esquematiza los resultados obtenidos al evaluar el parámetro de Linealidad del método tomando las 3 concentraciones como réplicas de la muestra y preparadas por duplicado, en cada una de ellas, con el siguiente resultado, aplicando la ecuación de la recta para la verificación de la confiabilidad del método.

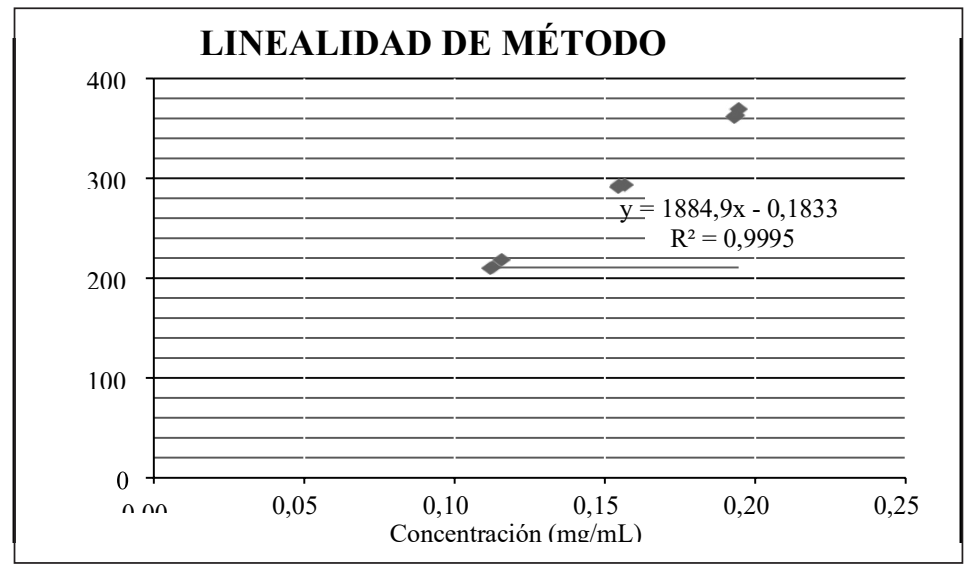

Figura2. Área versus concentración de linealidad del método de senósidos en tabletas de chocolate. 
En la tabla 4, se muestran los resultados de la evaluación del parámetro de Exactitud del método, para lo que se prepararon 3 concentraciones en el rango de trabajo establecido y luego de ello se analizaron los porcentajes de recuperación obtenidos para demostrar de esta forma si el método cumple con los criterios para demostrar su exactitud.

Tabla 4. Exactitud (recuperación) de senósidos en tabletas de chocolate.

\begin{tabular}{|c|c|c|c|c|c|c|}
\hline Concentración & Muestras & $\begin{array}{l}\text { Peso } \\
(\mathrm{mg})\end{array}$ & $\begin{array}{c}\text { Senósidos } \\
\text { añadido } \\
\text { (mg) }\end{array}$ & $\begin{array}{c}\text { Senósidos } \\
\text { hallado } \\
(\mathrm{mg})\end{array}$ & $\begin{array}{l}\text { Promedio } \\
\text { senósidos } \\
\text { hallado } \\
(\mathrm{mg})\end{array}$ & $\begin{array}{c}\text { Recuperación } \\
\%\end{array}$ \\
\hline \multirow{3}{*}{$75 \%$} & M1 & 18,72 & 11,55 & $\begin{array}{l}11,44 \\
11,43\end{array}$ & 11,44 & 99,00 \\
\hline & M2 & 18,13 & 11,19 & $\begin{array}{l}10,95 \\
11,04\end{array}$ & 11,00 & 98,29 \\
\hline & M3 & 18,23 & 11,25 & $\begin{array}{l}11,06 \\
11,03\end{array}$ & 11,04 & 98,17 \\
\hline \multirow{3}{*}{$100 \%$} & M1 & 25,01 & 15,43 & $\begin{array}{l}15,21 \\
15,29\end{array}$ & 15,25 & 98,82 \\
\hline & M2 & 25,05 & 15,46 & $\begin{array}{l}15,33 \\
15,35\end{array}$ & 15,34 & 99,24 \\
\hline & M3 & 25,34 & 15,63 & $\begin{array}{l}15,36 \\
15,35\end{array}$ & 15,36 & 98,24 \\
\hline \multirow{6}{*}{$125 \%$} & M1 & 31,51 & 19,44 & $\begin{array}{l}19,31 \\
19,30\end{array}$ & 19,31 & 99,30 \\
\hline & M2 & 31,37 & 19,36 & $\begin{array}{l}19,02 \\
19,02\end{array}$ & 19,02 & 98,26 \\
\hline & M3 & 31,28 & 19,30 & $\begin{array}{l}18,94 \\
18,93\end{array}$ & 18,93 & 98,10 \\
\hline & & & & & Promedio: & $98,60 \%$ \\
\hline & & & & \multicolumn{2}{|c|}{ Desviación estándar relativa: } & 0,0049 \\
\hline & & & & \multicolumn{2}{|c|}{ Coeficiente de variación (\%): } & $0,49 \%$ \\
\hline
\end{tabular}

En la tabla 5, se muestran los resultados de la evaluación del parámetro Precisión del método y para ello se realizaron los ensayos de repetibilidad de los resultados del análisis en las mismas condiciones de seis muestras y la precisión intermedia utilizando el mismo método realizado por diferentes analistas calificados. 
Tabla 5. Precisión: repetibilidad y precisión intermedia entre el analista uno y dos de senósidos en tabletas de chocolate.

\begin{tabular}{|c|c|c|c|c|c|c|}
\hline \multirow[b]{2}{*}{ Muestras } & \multicolumn{3}{|c|}{ Analista 1} & \multicolumn{3}{|c|}{ Analista 2} \\
\hline & $\begin{array}{c}\text { Senósidos } \\
\mathrm{mg} / \mathrm{tab}\end{array}$ & $\begin{array}{l}\text { Promedio } \\
\text { senósidos } \\
\text { mg/tab }\end{array}$ & $\%$ & $\begin{array}{c}\text { Senósidos } \\
\mathrm{mg} / \mathrm{tab}\end{array}$ & $\begin{array}{l}\text { Promedio } \\
\text { senósidos } \\
\text { mg/tab }\end{array}$ & $\%$ \\
\hline \multirow{2}{*}{ M1 } & 15,48 & \multirow{2}{*}{15,49} & \multirow{2}{*}{103,3} & 15,89 & \multirow{2}{*}{15,88} & \multirow{2}{*}{105,9} \\
\hline & 15,50 & & & 15,86 & & \\
\hline \multirow[t]{2}{*}{ M2 } & 15,36 & \multirow{2}{*}{15,34} & \multirow{2}{*}{102,3} & 15,63 & \multirow{2}{*}{15,61} & \multirow{2}{*}{104,0} \\
\hline & 15,33 & & & 15,59 & & \\
\hline \multirow{2}{*}{ M3 } & 15,30 & \multirow{2}{*}{15,30} & \multirow{2}{*}{102,0} & 15,46 & \multirow{2}{*}{15,48} & \multirow{2}{*}{103,2} \\
\hline & 15,30 & & & 15,50 & & \\
\hline \multirow{2}{*}{ M4 } & 15,61 & \multirow{2}{*}{15,60} & \multirow{2}{*}{104,0} & 15,57 & \multirow{2}{*}{15,67} & \multirow{2}{*}{104,5} \\
\hline & 15,59 & & & 15,77 & & \\
\hline \multirow{2}{*}{ M5 } & 15,58 & \multirow{2}{*}{15,58} & \multirow{2}{*}{103,9} & 15,58 & \multirow{2}{*}{15,51} & \multirow{2}{*}{103,4} \\
\hline & 15,58 & & & 15,44 & & \\
\hline \multirow[t]{8}{*}{ M6 } & 15,38 & \multirow{2}{*}{15,38} & \multirow{2}{*}{102,5} & 15,73 & \multirow{2}{*}{15,70} & \multirow{2}{*}{104,6} \\
\hline & 15,38 & & & 15,67 & & \\
\hline & & Promedio: & $103,0 \%$ & & Promedio: & $104,3 \%$ \\
\hline & & RSD: & 0,0082 & & RSD: & 0,0093 \\
\hline & & \multirow[t]{4}{*}{ C.V. $(\%)$ : } & \multirow[t]{4}{*}{$0,82 \%$} & & C.V. $(\%)$ & 0,93 \\
\hline & & & & Promed & nalista 1 y 2 : & $103,6 \%$ \\
\hline & & & & D del analis & y analista 2 : & 0,0106 \\
\hline & & & & o) del analis & y analista 2 : & $1,06 \%$ \\
\hline
\end{tabular}

C.V.: coeficiente de variación RSD.: desviación estándar relativa M: muestra

En la tabla 6, se muestran los resultados del parámetro de Robustez del método, evaluando una misma muestra en condiciones de almacenamiento de refrigeración después de 24 horas, analizados frente a estándares recientemente preparados en las mismas condiciones y así determinar si el método cumple con el parámetro requerido. 
Tabla6. Robustez entre análisis inicial y final de senósidos en tabletas de chocolate.

\begin{tabular}{|c|c|c|c|c|c|c|c|}
\hline \multirow[b]{2}{*}{ Muestra } & \multicolumn{3}{|c|}{ Inicio } & \multicolumn{3}{|c|}{$\begin{array}{c}\text { Final } \\
\text { (después de } 24 \text { horas refrigerado) }\end{array}$} & \multirow[b]{2}{*}{$\begin{array}{c}\text { Diferencia } \\
\text { ly1 - y0l }\end{array}$} \\
\hline & $\begin{array}{l}\text { Senósidos } \\
\mathrm{mg} / \mathrm{tab} \text {. }\end{array}$ & $\begin{array}{l}\text { Promedio } \\
\text { senósidos } \\
\text { mg/tab. }\end{array}$ & $\%(\mathbf{y} 0)$ & $\begin{array}{l}\text { Senósidos } \\
\mathrm{mg} / \mathrm{tab} .\end{array}$ & $\begin{array}{c}\text { Promedio } \\
\text { senósidos } \\
\text { mg/tab. }\end{array}$ & $\%(\mathbf{y 1})$ & \\
\hline M1 & $\begin{array}{l}15,48 \\
15,50\end{array}$ & 15,49 & 103,29 & $\begin{array}{l}15,51 \\
15,50\end{array}$ & 15,50 & 103,36 & 0,07 \\
\hline M2 & $\begin{array}{l}15,36 \\
15,33\end{array}$ & 15,34 & 102,29 & $\begin{array}{l}15,32 \\
15,35\end{array}$ & 15,34 & 102,25 & 0,05 \\
\hline \multirow[t]{8}{*}{ M3 } & $\begin{array}{l}15,30 \\
15,30\end{array}$ & 15,30 & 101,99 & $\begin{array}{l}15,35 \\
15,31\end{array}$ & 15,33 & 102,21 & 0,23 \\
\hline & Promedio & 15,38 & 102,52 & Promedio & 15,39 & 102,61 & \\
\hline & RSD & 0,0066 & 0,0066 & RSD & 0,0064 & 0,0064 & \\
\hline & C.V. $(\%)$ & $0,66 \%$ & $0,66 \%$ & C.V. $(\%)$ & $0,64 \%$ & $0,64 \%$ & \\
\hline & \multicolumn{6}{|c|}{ Promedio de inicio y final (después de 24 horas refrigerado): } & $102,56 \%$ \\
\hline & \multicolumn{6}{|c|}{ RSD de inicio y final (después de 24 horas refrigerado): } & 0,0058 \\
\hline & \multicolumn{6}{|c|}{ C.V. (\%) de inicio y final (después de 24 horas refrigerado): } & $0,58 \%$ \\
\hline & Diferencia c & promedio & e inicio & inal (despué & e 24 horas $r$ & rigerado): & 0,08 \\
\hline
\end{tabular}

C.V.: coeficiente de variación RSD.: desviación estándar relativa M: muestra

Referente a la linealidad, debe existir una relación lineal entre la señal analítica ( $y$ ) y la concentración $(x)$; en la figura 1 y figura 2, se grafican las curvas de calibración (relación de áreas versus concentración) para el sistema y para el método, en las que se puede apreciar la ecuación de la recta: para linealidad del sistema $y=3130,6 x+1,8289$ y para linealidad del método $y=1884,9 x-0,1833$. De acuerdo a la $\mathrm{AEFI}^{5}$, el valor para coeficiente de correlación es recomendable que sea mayor que 0,999 y para Gary ${ }^{10}$, el valor de coeficiente de determinación debe ser mayor que 0,998; lo cual indica un ajuste aceptable de los datos a la línea de regresión, en estas condiciones los resultados para linealidad del sistema $r=$ 0,9996 y $r 2=0,9991$ y para linealidad del método $r=0,9997$ y $r 2=0,9995$, con estos valores se puede decir que existe un alto grado de relación entre la variable $x$ (concentración) y la variable $y$ (área).

En la tabla 4, se muestra que el porcentaje de recuperación del parámetro de exactitud, que es de 98,60\%, presenta un Coeficiente de Variación de 0,49\%, siendo estos resultados lo recomendado por AEFI5, donde los criterios de aceptación para la recuperación deben estar entre los valores 98,0-102,0 \% y con un coeficiente de variación recomendado por USP 42 NF 3715 que debe ser menores a $2 \%$. 
Para el análisis del parámetro de robustez, se evaluó la estabilidad de la muestra en una condición, muestras después de 24 horas en refrigeración, donde se observó que las muestras no sufren cambios con el método analítico y no afectan la reproducibilidad de los resultados, siendo el porcentaje de Coeficiente de Variación C.V. (\%) de 0,58 \%.

\section{CONCLUSIONES}

Se logró validar un método analítico para cuantificar senósidos en tabletas de chocolate por cromatografía líquida de alta resolución (HPLC) con una buena separación, una pureza de pico cromatográfico de 99,99\% para el pico del estándar y 99,94 \% para el pico de la muestra, para lo cual se utilizó una columna cromatográfica de L11 (fenilo), 150 mm x 4,6 mm x $5 \mu \mathrm{m}$. Se estableció un tiempo de retención de 15 minutos, aproximadamente, para el pico principal, a una longitud de onda de $274 \mathrm{~nm}$, y con un volumen de inyección de $40 \mu \mathrm{L}$. La gradiente de flujo establecida oscila entre 0,4 a $1,5 \mathrm{~mL} / \mathrm{min}$. Se establecieron parámetros de validación de un método analítico por cromatografía líquida de alta resolución (HPLC), demostrando que es específico, después de determinar las interferencias una vez sometida a estrés. Es lineal en concentraciones de 0,$07 ; 0,11 ; 0,15 ; 0,19$ y $0,23 \mathrm{mg} / \mathrm{mL}$, con un coeficiente de correlación de 0,9996 . La precisión se determinó con una repetibilidad y precisión intermedia que tiene un Coeficiente de Variación (C.V.) menor al $2 \%$. La exactitud mostró un porcentaje de recuperación de $98,60 \%$ y la robustez con un coeficiente de variación de $0,58 \%$, después de 24 horas.

\section{REFERENCIAS BIBLIOGRÁFICAS}

1. The United States Pharmacopeia. USP 41. Rockville, Maryland: The United States Pharmacopeial Convention; 2018.

2. Validation of Analytical procedures:Text and Methodology Q2(R1). Geneva, Switzerland: International Conference on Harmonisation; 2005.

3. International Union of Pure and Applied Chemistry. Selectivity in Analytical Chemistry. Pure Appl Chem. 2001;73(8):1381-1386.

4. Castillo Aguilar B, González Hernández R. Protocolo de validación de métodos analíticos para la cuantificación de fármacos. Rev Cubana Farm. [Internet] 1997 [Citado 11 mayo 2021]; 30(1) Disponible en: http://scielo.sld.cu/scielo.php?script=sci arttext\&pid=S0034-75151996000100009

5. Asociación Española de Farmacéuticos de la Industria. Validación de Métodos Analíticos. Barcelona: Publicaciones AEFI; 2001.

6. Pacheco GJ. Evaluación de los niveles de los de Benzo[a] Pireno por Cromatografía Líquida de alta resolución (HPLC) en pollos. [Tesis de maestría]. Arequipa: Universidad Católica de Santa María; 2017. 
7. Samaniego Joaquín J, Arias Arroyo G. Desarrollo y Validación de una Metodología Analítica por HPLC para la Cuantificación Simultánea de Fenilefrina clorhidrato, Paracetamol, Salicilamida, Cafeína y Clorfenamina Maleato en tabletas. Rev Soc Quím Perú. 2016;82(2):196-207.

8. Skoog DA, West DM, Holler FJ, Crouch SR. Fundamentos de Química Analítica. Octava Edición. Madrid: Thomson Paraninfo; 2005.

9. Rubinson KA, Rubinson JF. Análisis Instrumental. Madrid: Pearson Educación S.A.; 2000 .

10. Christian GD. Química Analítica. Sexta Edición ed. México: McGraw-Hill/ Interamericana Editores, S. A; 2009.

11. Bavand Savadkouhi M, Vahidi H, Ayatollahi AM, Hooshfar S, Kobarfard F. RP-HPLC Method Development and Validation for Determination of Eptifibatide Acetate in Bulk Drug Substance and Pharmaceutical Dosage Forms. Iran J Pharm Res. 2017;16(2):490497.

12. Bor M, Guilarte A, Guzmán L, Macías K, Mendoza W. Validación de un método por RPHPLC para la determinación de Tiocolchicósido en tabletas. Afinidad 2017; 75(581):3238.

13. Funes JI, Meza MN, Ponce HD. Desarrollo y validación de un método para la cuantificación de atorvastatina en tabletas mediante HPLC-DAD. Portal de la Ciencia 2016; 9:27-41.

14. Guarniz Aguilar D. Validación de un método analítico por cromatografía líquida de alta resolución (HPLC) para la disolución de Ezetimiba $10 \mathrm{mg}$ y Simvastatina $40 \mathrm{mg}$ en tabletas. [Tesis grado de bachiller]. Trujillo: Universidad Nacional de Trujillo; 2017.

15. Ccorimanya Gutierrez K. Desarrollo y Validación Prospectiva de una técnica analítica por Cromatografía Líquida de alta performance (HPLC) para cuantificar Lidocaína Base en una pomada, Arequipa-2012. [Tesis de titulo de Químico Farmacéutico]. Arequipa: Universidad Católica de Santa María; 2012.

16. Enciso Rodríguez M. Validación de la técnica analítica por cromatografía líquida de alta performance (HPLC) para la determinación de fenilefrina clorhidrato, guaifenesina y dextrometorfano bromhidrato en jarabe. Lima -2013. [Tesis de titulo de Químico Farmacéutico]. Ayacucho: Universidad Nacional de San Cristóbal de Huamanga; 2014.

17. Heisler EV, Buda MLD, Schimith MD, Badke MR, Ceolin S, Heck RM. Uso de plantas medicinales en el cuidado de la salud: la producción científica de tesis y disertaciones de enfermería brasileña. Enferm Glob. 2015;14(39):390-403.

18. Gennaro AR. Remington Farmacia. 20 Edición ed. Buenos Aires: Editorial Medica Panamericana; 2003.

19. Mattos Gesteira E, Alonso Beato MT, Santos Bobillo MT, Miguel Ladero Álvarez M, Ladero Santos I. Plantas medicinales espanolas. Familia Leguminosae. Stud Bot. 2009; 28: 9-156. 